\title{
BMJ Open SAFETEL randomised controlled feasibility trial of a safety planning intervention with follow-up telephone contact to reduce suicidal behaviour: study protocol
}

\author{
Rory C O'Connor, ${ }^{1}$ Jenna-Marie Lundy, ${ }^{1}$ Corinna Stewart, ${ }^{1}$ Susie Smillie, ${ }^{2}$ \\ Heather McClelland, ${ }^{1}$ Suzy Syrett, ${ }^{1}$ Marcela Gavigan, ${ }^{2}$ Alex McConnachie, ${ }^{3}$ \\ Michael Smith, ${ }^{4}$ Daniel J Smith, ${ }^{5}$ Gregory K Brown, ${ }^{6}$ Barbara Stanley, ${ }^{7}$ \\ Sharon Anne Simpson ${ }^{2}$
}

To cite: O'Connor RC, Lundy J-M, Stewart C, et al. SAFETEL randomised controlled feasibility trial of a safety planning intervention with follow-up telephone contact to reduce suicidal behaviour: study protocol. BMJ Open 2019;9:e025591. doi:10.1136/ bmjopen-2018-025591

- Prepublication history and additional material for this paper are available online. To view these files, please visit the journal online (http://dx.doi org/10.1136/bmjopen-2018025591).

Received 23 July 2018 Revised 22 October 2018 Accepted 13 November 2018

Check for updates

(C) Author(s) (or their employer(s)) 2019. Re-use permitted under CC BY-NC. No commercial re-use. See rights and permissions. Published by BMJ.

For numbered affiliations see end of article.

Correspondence to

Professor Rory C O'Connor;

Rory.0Connor@glasgow.ac.uk

\section{ABSTRACT}

Introduction There are no evidence-based

interventions that can be administered in hospital settings following a general hospital admission after a suicide attempt.

Aim To determine whether a safety planning intervention (SPI) with follow-up telephone support (SAFETEL) is feasible and acceptable to patients admitted to UK hospitals following a suicide attempt.

Methods and analysis Three-phase development and feasibility study with embedded process evaluation. Phase I comprises tailoring an SPI with telephone follow-up originally designed for veterans in the USA, for use in the UK. Phase II involves piloting the intervention with patients $(n=30)$ who have been hospitalised following a suicide attempt. Phase III is a feasibility randomised controlled trial of 120 patients who have been hospitalised following a suicide attempt with a 6-month follow-up. Phase III participants will be recruited from across four National Health Service hospitals in Scotland and randomised to receive either the SPI with telephone follow-up and treatment as usual $(n=80)$ or treatment as usual only $(n=40)$. The primary outcomes are feasibility outcomes and include the acceptability of the intervention to participants and intervention staff, the feasibility of delivery in this setting, recruitment, retention and intervention adherence as well as the feasibility of collecting the self-harm readmission to hospital outcome data. Statistical analyses will include description of recruitment rates, intervention adherence/use, response rates and estimates of the primary outcome event rates, and intervention effect size (Phase III). Thematic analyses will be conducted on interview and focus group data.

Ethics and dissemination The East of Scotland Research Ethics Service (EoSRES) approved this study in March 2017 (GN17MH101 Ref: 17/ES/0036). The study results will be disseminated via peer-reviewed publication and conference presentations. A participant summary paper will also be disseminated to patients, service providers and policy makers alongside the main publication. Trial registration number ISRCTN62181241.

\section{Strengths and limitations of this study}

- SAFETEL will test the feasibility and acceptability of a safety planning intervention (SPI) with follow-up telephone support to patients admitted to UK hospitals following a suicide attempt.

- We have employed a collaborative person-centred approach to support the development of the SPI by involving those with lived experience as well as academics and clinicians.

- A process evaluation is embedded within the study.

- We have employed a mixed-methods approach (interviews, questionnaires, focus groups, medical records and hospital admission data).

- To enhance generalisability, this study is conducted in four hospitals.

\section{INTRODUCTION}

Suicide and self-harm are major public health problems. According to WHO, 804000 people die by suicide each year across the globe, ${ }^{1}$ with approximately 6000 people dying by suicide each year in the UK. Those with a history of self-harm are at a markedly increased risk of suicide $^{2}$; indeed $16 \%$ of those who are treated in hospital will have self-harmed again within 1 year and 1 in 25 patients will die by suicide within 5 years. ${ }^{3}$ Despite the increased risk of suicide, there is a lack of evidence-based interventions within general hospital settings for those who have attempted suicide specifically. Although there are challenges in determining suicidal intent and debate about definitions of self-harm, ${ }^{4}$ the majority of patients admitted to hospital following selfharm are cases of attempted suicide. ${ }^{5}$ Therefore, delivering effective treatment in hospital and by other means in the weeks following a 
suicide attempt represents a vitally important opportunity to mitigate future suicide risk.

Despite the fact that individuals who self-harm or attempt suicide represent a high-risk group for suicide, there is little research evidence about what works to reduce risk of future self-harm or suicide in this population. ${ }^{6-12}$ To date, there are no evidence-based interventions that can be administered in hospital following an emergency admission to reduce the risk of future suicidal behaviour in those who have attempted suicide. Existing interventions tend to be intensive and not delivered in acute settings. ${ }^{73-15}$ In general, patients in emergency department (ED) settings are 'assessed and referred on' for further care, ${ }^{16}$ although there is considerable variability.

This study addresses this evidence gap by seeking to answer the following research question; can a new, innovative, theory-driven safety planning intervention (SPI) with follow-up telephone support (SAFETEL), originally developed for use in veterans' hospitals in the USA, be tailored and made feasible for use with patients admitted to UK general hospitals following a suicide attempt?

\section{The SAFETEL intervention}

SAFETEL is an innovative and theoretically driven SPI with follow-up telephone support which was developed in the USA and aims to reduce suicide attempts. The SPI is a collaborative emergency safety plan developed by the patient in collaboration with a trained practitioner. The SPI is then supplemented with up to five structured follow-up telephone calls over 4 weeks.

A cohort comparison trial of suicidal ED patients in US veteran's hospitals ${ }^{17}$ found that SPI and phone follow-up reduced suicidal behaviours and increased treatment engagement in the intervention condition. Thus, the intervention is very promising, pointing to the potential positive impacts of the SAFETEL intervention. Although developments in the USA are encouraging, it is important to determine whether this intervention can be tailored to and is feasible and acceptable in a UK, non-veteran, ED/ acute care setting. Then, if shown to be feasible and acceptable, it should be rigorously assessed in a future definitive randomised controlled trial (RCT).

In addition to the personal distress, suicide attempts and suicide incur high economic costs, therefore any intervention that reduces these occurrences will yield considerable economic benefits. Each death by suicide in the UK is estimated to cost in excess of $£ 1370000^{18}$ and direct costs of self-harm range from $£ 1500$ per annum to $£ 3524$ for 6 months. ${ }^{18} 19$ Indeed, the overall annual cost of general hospital management of self-harm (for England) is estimated to be $£ 162$ million per year. ${ }^{20}$ The SAFETEL intervention has the potential, therefore, to fill an important gap in service provision with clear clinical impact and to reduce National Health Service (NHS)/societal costs. Although the focus will be on feasibility, we will also record readmission to hospital following self-harm ${ }^{[1]}$ (Self-harm is defined, consistent with the NICE guidance, as intentional self-poisoning or self-injury, irrespective of type of motive or the extent of suicidal intent.) in the subsequent 6 months following the index suicide attempt to inform effect size estimates for a full trial.

\section{Aim}

To determine whether SAFETEL is feasible and acceptable in a UK NHS context. The study has the following specific objectives:

1. To adapt/tailor an innovative SPI with follow-up telephone support for use within UK NHS hospital settings.

2. To investigate how participants engage with the intervention.

3. To assess feasibility and acceptability of the intervention.

4. To investigate trial recruitment, retention and other trial processes including data collection.

5. To explore the barriers and facilitators to intervention implementation.

6. To collect data on readmission to hospital following self-harm in the 6 months following the index suicide attempt to inform the sample size required for a full trial.

7. To further develop and test the logic model and theoretical basis of the intervention (see online supplementary appendix 1 for the proposed study logic model).

8. To assess whether an effectiveness trial is warranted.

\section{METHODS AND ANALYSIS}

\section{Study design}

This study follows the Medical Research Council (MRC) guidance for the development and evaluation of complex interventions. ${ }^{21}{ }^{22}$ The SAFETEL study is a three-phase development and feasibility trial of an SPI with follow-up telephone support (figure 1) with embedded process evaluation.

\section{Phase I}

In consultation with key stakeholders (patients and NHS staff), the existing SAFETEL intervention will be adapted for administration within a UK NHS context.

\section{Phase II}

Piloting of the intervention with approximately 30 patients who have been admitted to hospital following a suicide attempt.

\section{Phase III}

A feasibility RCT with 120 patients who have been admitted to hospital following a suicide attempt. Participants will be randomised to either the SPI with follow-up telephone support+treatment as usual $(\mathrm{n}=80)$ or treatment as usual only $(n=40)$.

We are adhering to protocol V.4 dated 26 April 2018. Any additional changes to the protocol will be reported to the Study Sponsor and receive appropriate approvals, as required.

\section{Patient and public involvement}

One of the study co-investigators (and co-author) is a service user and was involved in the development of the 


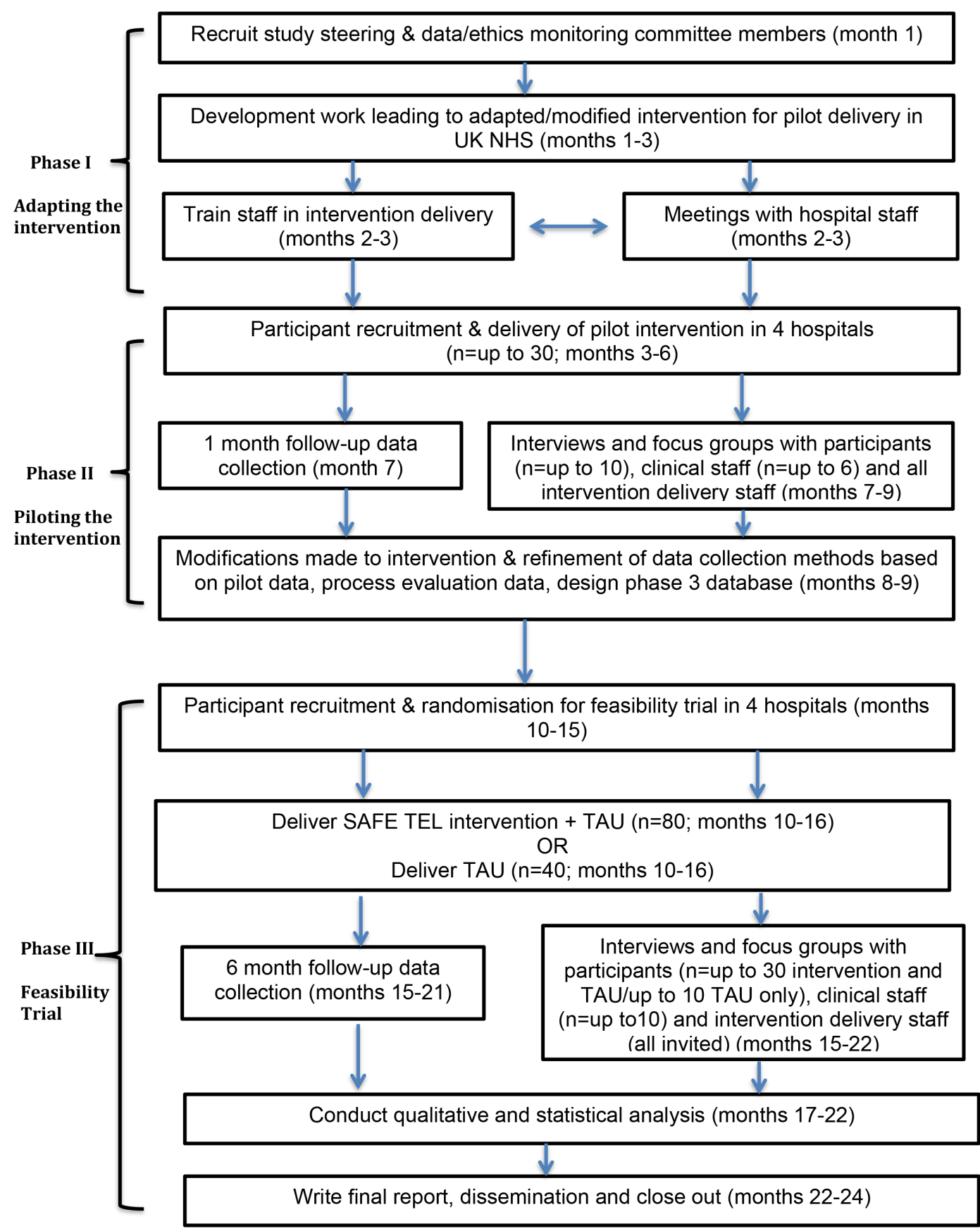

Figure 1 SAFETEL study flow diagram V.6. Month 1 begins 1 April 2017. NHS, National Health Service; TAU, treatment as usual.

research questions, the measures used and all aspects of study design and dissemination. As this is a feasibility study, we are seeking views from patients and others with experience of suicidal thoughts and attempts throughout.

\section{Settings}

Participants will be recruited from 4 NHS hospitals across two health boards in Scotland. SAFETEL will be delivered to intervention arm participants (in addition to treatment as usual) in these hospitals. The safety planning component of the intervention will be conducted faceto-face in these hospitals with telephone-based support sessions conducted up to 4 weeks later. The follow-up phone calls will typically begin when the participant has been discharged from hospital (see 'Follow-up telephone support' section for more details). Baseline data collection will also be conducted in the hospitals. Qualitative interviews and focus groups will be conducted at NHS or University of Glasgow sites, in phase III study participants will be given the option of being interviewed over the phone or in their own homes. Staff participating in this phase will be interviewed at their place of work or by telephone. 


\section{Participants}

To potentially receive the SAFETEL intervention, participants are eligible for the study if they meet the following criteria:

\section{Inclusion criteria}

1. Are aged 18 years or over.

2. Have been admitted to hospital presenting with a selfharm episode where there was evidence of suicidal intent (ie, a suicide attempt).

3. Have been assessed by the Liaison Psychiatry team.

4. Are proficient in English so that they can provide informed consent and complete written records in English.

\section{Exclusion criteria}

1. Indicate no suicidal intent.

2. Are medically unfit for interview.

3. Are unable to provide informed consent.

4. Have a level of English that is not sufficient to complete the assessment measures or SPI with follow-up telephone support.

5. Are participating in another psychological intervention study in the hospital.

6. Do not have access to a telephone.

The researcher will conduct a further assessment of the participant's eligibility in regard to presence of suicidal intent at the baseline assessment.

\section{Study procedures}

\section{Recruitment}

Phase I

Individuals with lived experience of suicide (ie, have been suicidal in the past) will be recruited by advertising via mental health organisations, websites and social media. Information about the study will be circulated at the hospital sites and clinical leads at the sites will be approached to be interviewed or to provide contact details of relevant staff to approach for interview.

\section{Phase II}

Liaison Psychiatry team staff at each hospital will be informed of the study and the participant inclusion and exclusion criteria. All team members will be asked to identify patients who are eligible for inclusion in the study (eg, present following self-harm episode where there was evidence of suicidal intent). The hospital staff will inform potential participants about the study and invite them to meet with the study researcher following their psychosocial assessment with the Liaison Psychiatry team. If the patient agrees, the researcher will approach the patient and provide them with the Participant Information Sheet, answer any questions and give them time to consider taking part. If the patient agrees, informed consent will be taken by the researcher and by consenting to take part, participants will agree to the research team accessing their medical notes. Research staff will confirm that participants meet inclusion criteria. We will also seek consent to audio-record the SPI for the purposes of fidelity monitoring of intervention delivery, but participation will not be contingent on consenting to this element. Similarly, information on the process evaluation interviews will be given and consent to future contact for this purpose will be sought, with participation in the intervention/study unaffected by opting not to consent to this element.

\section{Phase III}

Recruitment for phase III will be the same as per phase II (ie, referral following assessment by hospital Liaison Psychiatry teams) unless feedback from phase II suggests modifications. For phase III, however, participants will be informed that they will be randomised to receive either the SPI with follow-up telephone support+treatment as usual or treatment as usual only.

\section{Process evaluation}

Participants who consented to be contacted for this element of the study will be invited to participate in a one-to-one interview about their experiences of taking part. In phase II, participants will be contacted after they have finished the telephone support component of the intervention (approximately 1-2 months after baseline), and in phase III participants (both control and intervention arms) will be contacted approximately 6 months after baseline and once they have completed their involvement with the telephone follow-up component of the study. In phase II, the interview will be face-to-face at an NHS or University of Glasgow site, and in phase III, participants will be given the additional options of telephone interview or home visit. A process evaluation-specific Participant Information Sheet and Consent Form will be sent to all participants in advance of the interviews and reviewed at the interview to ensure it is understood and then the consent form will be completed. In the case of telephone interviews, verbal consent will be audio-recorded at the outset of the interview.

At phases II and III, NHS staff from the hospital sites and those directly involved in participants' care (eg, psychiatry liaison team members) will be invited to take part in interviews or focus groups using the same recruitment method as per phase I. The study research team will also be invited to participate in focus groups after the completion of phases II and III to discuss their experiences of delivering the intervention.

\section{Randomisation and blinding (phase III only)}

For phase III, participants will be randomised with a 2:1 ratio to receive either one of two study allocations: (i) the SPI with follow-up telephone support+treatment as usual or (ii) treatment as usual only. As we are most interested in exploring the feasibility of the intervention, we randomised 2:1 to extract the maximum information out of the data. Following consent and completion of the initial study measures, participants will be randomised using a telephone randomisation service provided by 
the Robertson Centre for Biostatistics (RCB), University of Glasgow (within the Glasgow Clinical Trials Unit). Neither the participant nor the researcher will know the allocation while initial study measures are being recorded. After randomisation, both the participant and the researcher will be unblinded to the participant's allocation, which is unavoidable given the nature of the trial. No changes in assignment will be possible. Randomisation will be performed using a mixed minimisation/ randomisation method. Within each hospital site, 3 out of every 15 participants will be allocated at random (in a 2:1 ratio), and 12 will be allocated according to a minimisation algorithm, designed to minimise imbalance with respect to hospital site, gender (as indicated by their current health record at date of consent) and history of self-harm (0-1 previous episodes vs 2 or more episodes). Whether participants are to be allocated at random, or by minimisation, will be determined by a computer-generated, block randomisation schedule, to be stored in a secure area of the RCB network, with access restricted to those responsible for the maintenance of the randomisation system.

Figure 2 shows the flow of participants through phase III of the study.

\section{Withdrawal, loss to follow-up and retention strategies}

Participants may fall into three categories relating to ceasing their participation in the study, these are:

i. Lost to completion of the SPI.

ii. Lost to follow-up data collection (ie, telephone follow-up calls).

iii. Withdrawn from the study.

Participants will be withdrawn from the study based on the following circumstances:

1. If the participant requests to be withdrawn from the study.

2. If it becomes known (eg, through telephone contact during the intervention or by other means) that the participant has lost capacity. (A person lacks capacity in relation to a matter if at the material time they are unable to make a decision for themselves in relation to the matter because of an impairment of, or a disturbance in the functioning of, the mind. This impairment may be permanent or temporary in nature.)

If a participant is withdrawn from the study, we will still use the assessment and other data collected (including follow-up clinical data regarding hospital readmission for self-harm) unless the participant explicitly states that they wish to have their data removed from the study.

\section{Study engagement, retention strategies and adverse events}

The study will use the following retention strategies to support participants to continue their engagement in the study alongside their treatment as usual commitments. The first telephone follow-up calls will be attempted up to 72 hours following discharge from hospital, and weekly thereafter at a time and date agreed with the participant. Call slots will be flexible and pragmatic as the study time elapses. In the event that a participant cannot be reached across three calls over two calendar days, the next call made will be to the participant's provided emergency contact to establish that the patient is safe and well. In the event that a follow-up call informs the research team directly or via a third party that the participant has been re-admitted to hospital for self-harm/suicide attempt (ie, the occurrence of an adverse event); or the call itself requires the study team to support the participant to seek help or to stay safe (ie, experiencing suicidal ideation), a further follow-up call will be offered. This additional call will act to provide adequate support to the participant and facilitate ongoing follow-up engagement. The researcher will follow the study Standard Operating Procedures to take appropriate action to maintain participant safety, which may include contacting existing care providers, referral to the ED or calling the emergency services. Data on the number, type and context of all adverse events will be routinely recorded in line with NHS and Good Clinical Practice regulations and reported to the Trial Steering Committee (TSC) and study senior management. The TSC will thereafter report to the study sponsor and governance management team as agreed in the initial stages of the ethical approval process. Given the nature of this study, we anticipate adverse events will occur.

\section{Control and intervention groups}

All participants will be invited to complete the study measures and to participate in the interviews for the process evaluation component of the study (regardless of the condition that they are allocated to).

\section{Control group}

Following randomisation, participants in the control group (ie, treatment as usual only) will be fully debriefed and will receive treatment as usual. Treatment as usual is variable but it may include referral to one of the following: (i) primary care; (ii) community psychiatric service; (iii) third sector service; (iv) specialist mental health service; (v) intensive home treatment; (vi) outpatient services; (vii) transfer to inpatient care; (viii) other services follow-up (ie, crisis card, social work input) or (ix) no further treatment plan. Treatment as usual will be characterised at each site as part of the process evaluation.

\section{Intervention group}

SATETEL will either be delivered by researchers trained by the intervention developers (Barbara Stanley and Gregory Brown), or by study investigators. This training will be cascaded down to new team members and researchers who support participant recruitment (ie, Mental Health Research Network researchers). The SPI element of the intervention will be delivered in the hospital before the patient is discharged. Within the SPI, patients are supported to complete a written, personalised safety plan in collaboration with the researcher. The safety plan comprises six steps outlined below. The purpose of the SPI is to help patients identify warning signs indicative 


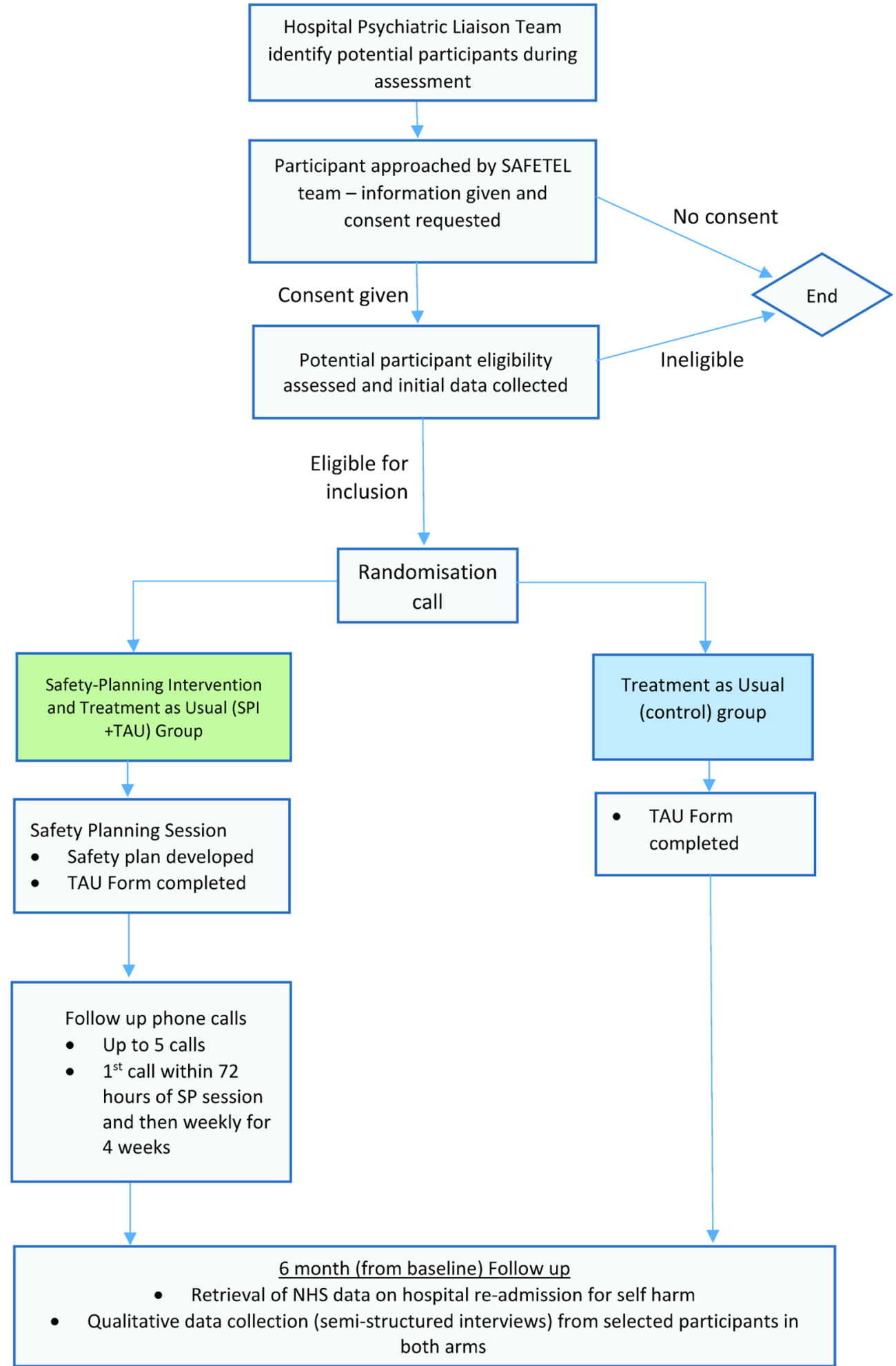

Figure 2 SAFETEL phase III participant flow diagram V.3. SPI, safety planning intervention; TAU, treatment as usual.

of an approaching suicidal crisis and to develop a list of internal coping strategies. In addition, patients also identify individuals in their social network who could provide distraction or support, and professional agencies who patients can contact during or preceding suicidal crises to reduce the risk of engaging in further suicidal behaviour. Although the order of completion of the safety plan is not completely fixed, participants will be encouraged to work through each step. Working through each step entails beginning with using one's internal resources, through to considering external resources such as calling a support person or professional service if they are in crisis and unable to keep themselves safe. They are also invited to take steps to make their environment safe by reducing access to lethal means (eg, restricting access to medication). 


\section{Developing the safety plan}

At the outset of the SPI collaboration, the researcher conducts a further risk assessment to ensure the participant is not at imminent risk of suicide. The patient is offered regular breaks during the assessment to mitigate fatigue and anticipated distress. Indeed, participants can become emotionally upset during safety plan completion, which is handled sensitively by the researcher (eg, offering to stop, take breaks, etc). The participant is also supported to complete the safety plan at a pace that suits their needs. The researcher explores the recent suicide attempt as a means to explain the purpose of the safety plan; and how to use it to support the participant to keep themselves safe during a suicidal crisis. This process aims to improve identification of warning signs that alert the participant that they may be approaching a crisis; explore the use of distraction techniques; encourage the idea of seeking social or professional support and restricting access to lethal means. When completing each step of the safety plan, the researcher explores the suitability and likelihood of employing these strategies during a suicidal crisis as well as providing examples of such strategies.

\section{Follow-up telephone support}

This component of the intervention consists of five structured telephone contacts with the participant over a period of 4 weeks. The first contact is typically delivered as soon as possible after discharge from hospital following the index suicide attempt (between 24 and 72 hours) followed by 4 weekly telephone contacts. The follow-up telephone calls comprise three components: 1) suicide risk assessment and mood check; 2) review of the participant's safety plan, with revisions made if required and 3) supporting treatment engagement through exploration of barriers to engagement, motivational enhancement, problem-solving and support. The duration of follow-up calls will vary but it is expected that they will last around $15 \mathrm{~min}$ on average. At the end of each follow-up call (apart from the final one), the participant is asked if they consent to another follow-up call. Follow-up telephone support is discontinued after five phone calls, if the participant no longer wishes to be contacted or if the participant can no longer be contacted. The researcher will attempt to contact the participant up to three times per scheduled contact point. They will send a text or leave a voicemail message if the participant cannot be reached by telephone and if the participant has consented to this. The researcher may also attempt to contact the participant by letter. In the event where a participant cannot be reached and there is concern regarding their safety, the researcher may contact the participant's emergency contact (recorded at the initial assessment with the participant's consent) or professional services involved in the patient's care (eg, their general practitioner (GP)). On the final follow-up call, in addition to the standard procedure, the participant is asked if they are still happy to be contacted for information regarding the process evaluation element of the study.

\section{Process evaluation measures}

The process evaluation will seek to assess feasibility and acceptability and explore the ways in which SAFETEL may operate to produce outcomes. Specifically, it will focus on intervention fidelity, exposure, reach, context, recruitment, retention and contamination, as well as the acceptability of study procedures. Table 1 presents the process evaluation framework and shows the various time points at which data collection and analysis are intended.

\section{Intervention fidelity checking}

With participants' consent, all SPI sessions in phases II and III will be audio-recorded. Fidelity of intervention delivery is being checked in different ways for the face-toface sessions and telephone sessions. For the face-to-face sessions, $20 \%$ of the recordings will be randomly selected to check fidelity against a standardised measure of fidelity for the SPI (SPI Rating Scale; Brown, G. K. \& Stanley, B. Safety Plan Intervention Rating Scale). These will be double coded by another team member and tested using Cronbach's $\alpha$ to test inter-rater reliability. A standardised checklist for the follow-up telephone support calls will be completed by the research team to enhance intervention fidelity and the results will be reported descriptively.

\section{Qualitative interviews with study participants}

We will conduct semi-structured interviews with intervention participants at phase II ( $\mathrm{n}=\mathrm{up}$ to 10$)$ and Phase III $(n=u p$ to 30$)$. Actual numbers will depend on data saturation. Participants will be purposively sampled based on a number of criteria (ie, gender, age, hospital site of recruitment, engagement with the intervention and history of self-harm). Semi-structured interview topic guides will be used and interviews will seek to explore participants' experience of the study and intervention including contextual factors, acceptability of study and intervention procedures, barriers and facilitators to engagement with the intervention and potential mediators of change. We will also seek to interview participants in the control arm at phase III ( $n=u p$ to 10) to explore their experiences of their treatment as usual, potential contamination and the acceptability of study procedures.

\section{Qualitative interviews/focus groups with staff}

We will conduct semi-structured interviews with NHS clinical staff involved in the care of patients who have been admitted to hospital following a suicide attempt at phase II ( $\mathrm{n}=$ up to 6$)$ and phase III ( $\mathrm{n}=\mathrm{up}$ to 10$)$. The interviews will focus on current context, procedures and services available to patients, feasibility of the intervention and acceptability of the study and intervention procedures, including experienced or perceived barriers and facilitators, intervention 'fit' within the setting and suggestions for improvement. In addition, we will conduct focus groups at phases II and III with researchers responsible for study recruitment, data collection and intervention delivery (all researchers will be included where possible). Focus groups will explore experiences of recruitment, 
Table 1 Process evaluation framework for analysis (V.3.0, 27 June 2018)

\section{Evaluation area}

Fidelity

(the degree to which the intervention was delivered as intended)

\section{Exposure}

(the extent to which participants received and understood the different elements of the intervention and whether they implemented these as intended. Their satisfaction with the intervention and barriers to receipt and implementation were also considered)

\section{Questions}

What is the intervention?

- Was the intervention (safety plan (SP) and follow-up telephone calls) delivered as intended?

Was there consistency in terms of how the intervention was delivered?

What, if any, adaptations were needed to the planned intervention? And were they needed?

What barriers, if any, were there to delivering the intervention in a consistent way? (SP and followup telephone calls)?

- To what extent did participants take up all potential elements of the full programme of intervention (SP and five follow-up telephone calls)?

- To what degree did participants receive the minimum dose (SP and one follow-up phone call)?

- To what extent was the SP completed as intended by the participant? If it was not what were the reasons for that?

- How did participants use the safety plan they had developed? (eg, frequency of use, practicalitywhere did they keep it, did they share with others)

- To what extent did participants alter or amend their safety plans throughout the course of the intervention?

- What elements of the intervention did participants find helpful/unhelpful and why? What elements of the intervention would participants change and why?

- What changes, if any, did participants feel that they implemented as a result of taking part in the intervention?

- What factors were involved in ongoing engagement with the intervention?

- What do participants report were barriers and facilitators to developing the SP, engaging with telephone support and using the SP in practice?

- What feedback do participants have regarding feasibility and acceptability of the SP and follow-up telephone calls?

\section{Reach \\ (the extent to which the target audience is reached by the intervention, as well as any 'spill over' effects on people not recruited)}

\section{Context}

(includes information relating to aspects of the context in which the intervention was delivered, as well as broader context that both practitioner and client were operating within that may influence intervention effectiveness)
How well does the study sample represent the population of interest?

- Did participants report sharing their SP with family or friends?

- To what extent did the intervention reach and influence people other than recruited participants?

- What participant-centred contextual factors influenced engagement with the intervention (safety planning and follow-up calls) and use of the SP in practice?

- What contextual factors within participants' day-to-day environment influenced engagement with the intervention (safety planning and follow-up calls) and use of the SP in practice?

- How did the context in which the intervention was delivered influence engagement with the intervention and use of the SP in practice?

- Was the SP useful in certain circumstances and not in others?

- How does the intervention fit in with what is delivered in hospital (how easy was is it to deliver in this setting and does it conflict with anything)?

-What were the particular context-related difficulties/issues that arose during the study in delivering the intervention?

Recruitment and retention
How did participants feel about being approached/recruited in hospital setting?
What motivated study participants to agree to take part? (And what kept them engaged?)
Were there any difficulties in recruitment?
What is the attrition rate overall and by subgroup? That is, intervention groups and control
What were the reasons for withdrawal?
Contamination
What are the characteristics of other groups or services people are attending or resources they are
Have participants used an SP or similar in the past?
Hid participants in the treatment as usual (TAU) arm investigate 'SP' strategy on their own?
Have any of the TAU arm participants seen intervention content from other participants?
How did randomisation to the TAU arm affect participants?

data collection and intervention delivery, as well as perception of participants' experiences.

\section{Data on recruitment, retention and adherence}

Data on the number of potential participants approached, who declined, were ineligible and those who consented and were retained will be recorded and presented in the
Consolidated Standards of Reporting Trials diagram for the study. Concerning adherence to the intervention, we will record details of all intervention-related contacts, including number/length of sessions of support completed and contact attempts made, in order to build a comprehensive picture of how participants engage with the study and the intervention. Data on the rate of safety plan completion 
and the use of safety plans between telephone contacts are recorded as well as the amount/and type of changes made to safety plans over the course of the (up to) five follow-up calls.

\section{Outcome measure feasibility \\ Baseline}

For phases II and III, all participants will be asked to complete a number of measures during the initial assessment at baseline with a trained researcher (see below). The purpose of collecting these will be to assess feasibility and acceptability of using these questionnaires in a full trial, as well as to characterise the sample and explore potential moderators. We will also record participant demographics, information on treatment as usual received by participants and other relevant information regarding the sample (eg, suicidal history), which may be considered potential moderators or mediators in a full trial. The schedule of baseline measure completion for phases II and III is outlined in figure 2 and detailed below:

- The Columbia Suicide Severity Rating Scale ${ }^{23}$ is a 20 -item semi-structured brief, valid and reliable tool used to assess suicide risk and suicidal ideation and behaviours, such as previous suicide attempts as well as interrupted and aborted attempts and preparatory behaviours.

- The Entrapment Scale $e^{24}$ is a 16 -item scale that examines feelings of entrapment and defeat using a 5-point Likert-type scale (ranging from 0 'not at all like me' to 4 'extremely like me'). It comprises two subscales: internal (10 items) and external entrapment (6 items).

- The Interpersonal Needs Questionnaire ${ }^{25}$ is a 12-item measure of perceived burdensomeness ( 7 items) and thwarted belongingness ( 5 items), with items rated on a 7-point Likert-type scale (ranging from 1 'not at all true for me' to 7 'very true for me').

- The ENRICHD Social Support Instrument ${ }^{26}$ is a 7-item measure that assesses four attributes of social support: emotional, instrumental, informational and appraisal on a 5-point Likert-type scale (ranging from 1 'none of the time' to 5 'all of the time').

- The following measure will also be completed at phase III only:

- The Suicide-Related Coping Scale ${ }^{27}$ (phase III only) is a 17-item measure that assesses suicide-related coping. It comprises two subscales: external coping, with items relating to recognising and using social support and professional resources during suicidal crisis and lethal means restriction, and internal coping, with items pertaining to self-administered coping strategies and confidence in relation to coping with suicidal feelings. Responses are rated on a 5-point Likert-type scale (ranging from 0 'strongly disagree' to 4 'strongly agree').

\section{Follow-up telephone calls}

Measures completed at follow-up calls include: (i) the contact inventory (ie, call duration, time between calls and number and means of participant contact); (ii) a mood and suicidal thoughts and behaviours assessment; (iii) a review of the safety plans and use; (iv) treatment engagement and (v) participant agreement to receive the next follow-up call.

\section{Six-month post-index data capture}

Follow-up data on hospital readmissions for self-harm after baseline will be collected at phase II (1-2 months postindex suicide attempt) and phase III (6 months postindex suicide attempt) using NHS clinical databases in order to assess the viability of collecting these data in a full trial.

\section{Data analysis}

The RCB within the University of Glasgow will provide statistical services in support of phase III of the trial. The RCB is part of the Glasgow Clinical Trials Unit, and has extensive experience of the design, analysis and reporting of clinical trials and epidemiological studies.

\section{Sample size}

It is estimated that Liaison Psychiatry teams across the four hospitals annually see at least 3700 patients who selfharm, and we estimate that $75 \%$ report suicidal intent. ${ }^{528}$ Therefore, across 6 months of recruitment for phase III, there will be approximately 1388 eligible participants; so we are aiming to recruit 20 participants per month. A sample of 120 participants is sufficient to explore the feasibility and acceptability of the intervention and allow estimation of the outcome event rates for a full trial.

\section{Quantitative data}

Statistical analysis will include descriptive summaries of recruitment rates, attrition and retention and intervention adherence. The baseline characteristics of the sample will be summarised. The primary outcome (readmission to hospital following self-harm within 6 months of the index suicide attempt) will be summarised by randomised group, and the intervention effect estimated using logistic regression, adjusting for minimisation factors (ie, hospital site, gender and history of self-harm). Associations between baseline characteristics and the primary outcome will also be assessed to explore potential moderators for a full RCT.

\section{Qualitative data}

Qualitative data from the interviews and focus groups conducted with study participants, NHS staff and the researchers who will deliver the intervention will be analysed via thematic analysis (phases II and III) using the approach by Braun and Clark. ${ }^{29}$ Thematic analysis is a systematic approach in which the data are initially coded and then collated into themes, which are then analysed in more detail to map out the overall data and examine relationships between them. Finally, themes are refined to produce an overall story of participants' views and experiences. Data collection and analysis of interview data will be conducted simultaneously and the 
analyses will inform data collection in terms of changes to the interview schedule (eg, adding new questions to probe particular areas of interest). Data collection will continue until data saturation is reached and variables coded using NVivo V.11.4.1. Due to the primary focus of the semi-structured interviews and focus groups, the identified themes are likely to be on areas of interest to the study evaluation (eg, recruitment, retention, acceptability, adherence, etc), but this method also allows unexpected themes to emerge and to be added to the coding framework. The coding framework will be discussed and refined with the other members of the study team. Twenty per cent of the interviews will be double coded to ensure reliability. Disagreements will be resolved by discussion.

Qualitative data will also be triangulated with quantitative data. We will draw on qualitative and quantitative data to test the logic model (see online supplementary appendix 1) and investigate mechanisms through which the intervention may operate in order to further develop the intervention theory (phase III). All analyses will be specified in a detailed Qualitative Analyses Plan and Process Evaluation Framework.

\section{Progression criteria from feasibility to full trial}

The feasibility and acceptability of both the trial methods and the SAFETEL intervention, and the potential for these to be developed and delivered in a full RCT are the key outcomes of this trial. These will be assessed using the progression criteria outlined in online supplementary appendix 2. These criteria have been developed by our Trial Management Group and approved by our TSC; who will also undertake final assessment of these following analyses of the findings of the study.

\section{DATA MANAGEMENT AND ACCESS}

The RCB (part of the Glasgow Clinical Trials Unit), within the University of Glasgow, will provide data management services in support of phase III of the trial. The RCB will create the database for phase III data, and provide the online electronic Case Record Form, as well as training in the use of the system. Data will be entered locally with data validation checks built in. RCB will also run routine data validation checks and alert the study management team to general issues or specific data queries.

All personal information will be encrypted and visible only to the research team; all personally identifiable information will be held separately from research data. The RCB statisticians will develop analysis programmes during the trial and communicate any data anomalies to RCB data managers. At the end of the trial, final data validation checks will be carried out prior to database lock. The study database will be held by RCB for the duration of the study and for a minimum of 5 years after study completion.

\section{Data sharing}

At baseline, potential participants are asked to consent to the following in order to participate in the study:

- Permission for the research team to access routinely collected medical data (including Community Health Indexnumbers) to determine what contact the participant has had with clinical services within 5 years of taking part in this study, where it is relevant to their taking part in the research.

- Permission for authorised representatives of the study Sponsor, NHS Greater Glasgow and Clyde/NHS Lothian and regulatory authorities to have access to their personal information and research data for the purposes of audit.

- In addition, participants are given the option of consenting to the research team sharing their data in the following circumstances:

- Anonymous storage of data in the UK data archive where other researchers can have access to this data only if they have scientific and ethical approval, and agree to preserve the confidentiality of this information as set out in the study consent form.

- Informing the participant's own GP and other relevant mental health professionals involved in their care, of their participation in the study and sending them a copy of the participant's safety plan.

\section{Serious adverse events}

As discussed in 'Study engagement, retention strategies and adverse events' section in detail; in the event of serious adverse events occurring within the study, standard operating procedures, robust recording and reporting measures to detail these occurrences will be employed. Any complaints made by participants or relevant adverse events will be recorded and reported to TSC. The TSC will thereafter report to the study sponsor and governance management team as agreed in the initial stages of the approval process. The TSC will take on the role of Data Monitoring Committee for oversight of adverse events. The TSC will comprise individuals with extensive expertise in clinical trials, suicide prevention research, biostatistics and clinical practice as well as lived experience.

\section{ETHICS AND DISSEMINATION}

The study will be conducted in accordance with the recommendations for physicians involved in research on human participants adopted by the 18th World Medical Assembly, Helsinki 1964 and later revisions. The study results will be disseminated via peer-reviewed publication and conference presentations. A participant summary paper will also be disseminated to patients and policy makers who wish to receive it alongside the main publication. 


\section{Trial status}

This trial is ongoing but all participants have now been randomised.

\section{Author affiliations}

${ }^{1}$ Suicidal Behaviour Research Laboratory, Institute of Health and Wellbeing,

University of Glasgow, Glasgow, UK

${ }^{2}$ Institute of Health and Wellbeing, MRC/CSO Social and Public Health Sciences Unit, University of Glasgow, Glasgow, UK

${ }^{3}$ Institute of Health and Wellbeing, Robertson Centre for Biostatistics, University of Glasgow, Glasgow, UK

${ }^{4}$ Mental Health Services, NHS Greater Glasgow and Clyde, Glasgow, UK

${ }^{5}$ Institute of Health and Wellbeing, University of Glasgow, Glasgow, UK

${ }^{6}$ Perelman School of Medicine, University of Pennsylvania, Philadelphia,

Pennsylvania, USA

${ }^{7}$ Department of Psychiatry, Columbia University College of Physicians and Surgeons, New York, New York, USA

Contributors ROC is principal investigator who together with SAS developed the study protocol alongside the other co-investigators (BS, GKB, MS, DJS, AMC and SSy), and has overall responsibility for the management of the trial. MG is the trial manager and is responsible for coordinating the trial. The Research Associate $\mathrm{J}-\mathrm{ML}$ also coordinates the trial and provides day-to-day management of the research team and oversees recruitment at hospital sites as well as data capture, supported by MG. SSm is the process evaluation researcher. MG, J-ML, CS and SSm were involved in finalising the study protocol, implementing study processes and drafting the manuscript. HMC and CS are research assistants on the trial and have responsibility for participant recruitment, data capture, contributing to the study design, reviewing the manuscript. AMC was involved in finalising the study protocol, in particular the statistical analyses, and reviewing the manuscript. BS and GKB had responsibility for training the research staff in delivering the intervention. SSy is the SAFETEL study peer researcher who is also responsible for representing patient views and focus group activity and reviewed the manuscript. All authors commented on and approved the final version of the manuscript.

Funding This research is funded by MQ Research (MQ16PI100009). SAS is supported by a UK Medical Research Council Strategic Award MC-PC-13027 and Medical Research Council and Chief Scientist Office core funding as part of the MRC/CSO Social and Public Health Sciences Unit 'Social Relationships and Health Improvement' programme (MC_UU_12017/11 and SPHSU11) and 'Complexity in Health Improvement' programme (MC_UU_12017/14 and SPHSU14).

Competing interests None declared.

Patient consent for publication Not required.

Ethics approval The East of Scotland Research Ethics Service approved this study in March 2017 (GN17MH101 Ref: 17/ES/0036).

Provenance and peer review Not commissioned; externally peer reviewed.

Open access This is an open access article distributed in accordance with the Creative Commons Attribution Non Commercial (CC BY-NC 4.0) license, which permits others to distribute, remix, adapt, build upon this work non-commercially, and license their derivative works on different terms, provided the original work is properly cited, appropriate credit is given, any changes made indicated, and the use is non-commercial. See: http://creativecommons.org/licenses/by-nc/4.0/.

\section{REFERENCES}

1. WHO. Preventing suicide: a global imperative. Geneva: WHO, 2014.

2. Chan MK, Bhatti H, Meader N, et al. Predicting suicide following self-harm: systematic review of risk factors and risk scales. $\mathrm{Br} \mathrm{J}$ Psychiatry 2016;209:277-83.

3. Carroll R, Metcalfe C, Gunnell D. Hospital presenting self-harm and risk of fatal and non-fatal repetition: systematic review and metaanalysis. PLoS One 2014;9:e89944.

4. Kapur N, Cooper J, O'Connor RC, et al. Non-suicidal self-injury v. attempted suicide: new diagnosis or false dichotomy? $\mathrm{Br} J$ Psychiatry 2013;202:326-8.
5. O'Connor RC, O'Carroll RE, Ryan C, et al. Self-regulation of unattainable goals in suicide attempters: a two year prospective study. J Affect Disord 2012;142:248-55.

6. Armitage CJ, Rahim WA, Rowe R, et al. An exploratory randomised trial of a simple, brief psychological intervention to reduce subsequent suicidal ideation and behaviour in patients admitted to hospital for self-harm. Br J Psychiatry 2016;208:470-6.

7. Gysin-Maillart A, Schwab S, Soravia L, et al. A novel brief therapy for patients who attempt suicide: a 24-months follow-up randomized controlled study of the Attempted Suicide Short Intervention Program (ASSIP). PLoS Med 2016;13:e1001968.

8. NICE. National collaborating centre for mental health (UK). British Psychological Society 2012:1332011.

9. O'Connor RC, Ferguson E, Scott F, et al. A brief psychological intervention to reduce repetition of self-harm in patients admitted to hospital following a suicide attempt: a randomised controlled trial. Lancet Psychiatry 2017;4:451-60.

10. O'Connor RC, Kirtley OJ. The integrated motivational-volitional model of suicidal behaviour. Philos Trans R Soc Lond B Biol Sci 2018;373:20170268

11. O'Connor RC, Nock MK. The psychology of suicidal behaviour. Lancet Psychiatry 2014;1:73-85.

12. Turecki G, Brent DA. Suicide and suicidal behaviour. Lancet 2016;387:1227-39.

13. Brown GK, Ten Have T, Henriques GR, et al. Cognitive therapy for the prevention of suicide attempts: a randomized controlled trial. JAMA 2005;294:563-70.

14. Brown GK, Jager-Hyman S. Evidence-based psychotherapies for suicide prevention: future directions. Am J Prev Med 2014;47:S186-S94.

15. Linehan MM, Comtois KA, Murray AM, et al. Two-year randomized controlled trial and follow-up of dialectical behavior therapy vs therapy by experts for suicidal behaviors and borderline personality disorder. Arch Gen Psychiatry 2006;63:757-66.

16. Stanley B, Brown GK. Safety planning intervention: a brief intervention to mitigate suicide risk. Cogn Behav Pract 2012:19:256-64.

17. Stanley B, Brown GK, Brenner LA, et al. Comparison of the safety planning intervention with follow-up vs usual care of suicidal patients treated in the emergency department. JAMA Psychiatry 2018;75:894.

18. McDaid D. Making an economic case for investing in suicide prevention Quo Vadis? In: O"Connor RC, Pirkis J, eds. International handbook of suicide prevention. 2nd ed. Chichester: Wiley Blackwell, 2016:775-90.

19. Sinclair JM, Gray A, Rivero-Arias O, et al. Healthcare and social services resource use and costs of self-harm patients. Soc Psychiatry Psychiatr Epidemiol 2011;46:263-71.

20. Tsiachristas A, McDaid D, Casey D, et al. General hospital costs in England of medical and psychiatric care for patients who self-harm: a retrospective analysis. Lancet Psychiatry 2017;4:759-67.

21. Craig P, Dieppe P, Macintyre S, et al. Developing and evaluating complex interventions: the new Medical Research Council guidance. Int J Nurs Stud 2013;50:587-92.

22. Moore GF, Audrey S, Barker M, et al. Process evaluation of complex interventions: Medical Research Council guidance. $B M J$ 2015;350:h1258.

23. Posner K, Brown GK, Stanley B, et al. The columbia-suicide severity rating scale: initial validity and internal consistency findings from three multisite studies with adolescents and adults. Am J Psychiatry 2011;168:1266-77

24. Gilbert P, Allan S. The role of defeat and entrapment (arrested flight) in depression: an exploration of an evolutionary view. Psychol Med 1998;28:585-98.

25. Van Orden KA, Cukrowicz KC, Witte TK, et al. Thwarted belongingness and perceived burdensomeness: construct validity and psychometric properties of the Interpersonal Needs Questionnaire. Psychol Assess 2012;24:197-215.

26. Vaglio J, Conard M, Poston WS, et al. Testing the performance of the ENRICHD Social Support Instrument in cardiac patients. Health Qual Life Outcomes 2004;2:24.

27. Stanley B, Green KL, Ghahramanlou-Holloway M, et al. The construct and measurement of suicide-related coping. Psychiatry Res 2017;258:189-93.

28. O'Connor RC, Smyth R, Williams JM. Intrapersonal positive future thinking predicts repeat suicide attempts in hospital-treated suicide attempters. J Consult Clin Psychol 2015;83:169-76.

29. Braun V, Clarke V. Using thematic analysis in psychology. Qual Res Psychol 2006;3:77-101. 